(RESEARCH ARTICLE)

\title{
A novel suturing technique, based on physical principles
}

\author{
FJ Pérez Lara $1{ }^{1}{ }^{*}$, R Zubizarreta Jimenez ${ }^{2}$, JM Hernández González ${ }^{3}$, T Prieto-Puga ${ }^{3}$ and F Moya Donoso ${ }^{3}$ \\ ${ }^{1}$ Chief of Surgery Service. Hospital de Antequera. Málaga. \\ ${ }^{2}$ CEO in DOXA microfluidics (mechanical engineer). \\ ${ }^{3}$ Digestive Surgeon. Service of Surgery. Hospital de Antequera. Málaga.
}

Publication history: Received on 20 November 2020; revised on 28 November 2020; accepted on 02 December 2020

Article DOI: https://doi.org/10.30574/wjarr.2020.8.3.0462

\begin{abstract}
Introduction: In medicine, sutures have been used for thousands of years to close wounds. However, despite the great advances that have been made in technology, little progress has taken place in suturing techniques and most surgeons continue to use the same two classical techniques (continuous suture or interrupted suture). This paper proposes a suturing technique ("double diabolo") in which a pattern of eight 45-degree angles is formed, in the view that this achieves a notable reduction in the tension exerted ( $30 \%$ at each angle), thereby increasing the overall strength of the suture.
\end{abstract}

Material and Method: In this study, we compare the physical tensions exerted on the suture thread and its entry/exit points, on the one hand by the two techniques most often used - interrupted suture (Type 1) and continuous suture (Type 2) - and on the other, by the proposed "double-diabolo" suture (Type 3).

Results: The Type 3 suturing method produced the lowest levels of tension on the thread, almost $65 \%$ less than those of Type 1 and $50 \%$ less than those of Type 2 . The tension on the wound was also considerably lower, with reductions of $33 \%$ and $50 \%$ in comparison with Types 1 and 2, respectively.

Conclusions: The results obtained in this study corroborate our hypothesis that the proposed double-diameter suture method exerts less tension on the thread and on the entry/exit points than conventional techniques. Theoretically it would be recommended for cases in which the suture must withstand significant tension as laparotomy closure

Keywords: Suture; Technique; Physical Principles; Tension

\section{Introduction}

Although sutures have been used to close wounds throughout recorded history [1, 2], in recent years there have been major technological advances in this field. Many clinical and experimental studies, in fields including physics, chemistry and the study of biomaterials, have been made to obtain suture threads that are more resistant and more elastic, with better characteristics, and surgical needles that are optimised according to the type of wound tissue presented [3-6].

However, while the materials used have improved greatly, suturing techniques have remained virtually unchanged. Thus, the same two basic techniques have been used since suturing was first employed to close a wound: interrupted sutures (in which the stitches are not connected) and continuous sutures (in which they are). Neither of these approaches (nor the minor variations that have been introduced) address the question of achieving an optimum pattern of stitching so that the surgical thread and the wound tissues are required to withstand the least possible tension, thus minimizing the possibility of breakage and tearing, respectively.

\footnotetext{
* Corresponding author: Francisco Javier Pérez Lara

Hospital de Antequera. Secretaría de Cirugía (3o planta). Avenida Poeta Muñoz Rojas s/n. Antequera. Málaga. 
With the current understanding of physical forces, all the forces exerted on a thread fixed at two points can be described according to parameters such as direction, angulation and the intersection of threads... However, to our knowledge no research has yet been undertaken to determine how these physical laws can be applied to minimise the tension exerted on a surgical suture. Accordingly, we propose to apply the laws of physics to identify the ideal suture pattern, i.e. one that minimises the tension exerted on each suture thread and on the entry/exit points.

The tension exerted on a suture thread varies directly with the angle of approach. Therefore, we propose a suture method ("double-diabolo") in which each point is supported by two central inverted double angles and by four lateral angles, thus creating eight 45-degree angles (Figure 1) distributing the tension that the suture must withstand.

We hypothesise that with this "double-diabolo" suture technique both the suture thread and the entry/exit points are subjected to less tension (for a given separation force) than is the case with conventional sutures and therefore are more resistant to breakage and tearing, respectively.

\section{Material and methods}

In this study, in terms of the physical forces involved, we compare the two suturing techniques commonly used, i.e. interrupted (Type 1) and continuous sutures (Type 2), with an alternative approach, that of the "double-diabolo" suture (Type 3).

This publication constitutes the first phase of a three-phase study of the above-mentioned suture groups. In this first phase, a theoretical physical model is applied to determine the tension that would be exerted on the suture thread and entry/exit points with each type of suture, when an identical force is exerted in each case. In the second phase of the study, the results obtained from the first phase will be transferred to the laboratory to examine whether these theoretical postulates are met. In the third and final phase, we will compare the three types of suture in clinical practice.

\subsection{Interrupted sutures (Type 1)}

This method consists of creating stitches that are discrete and perpendicular to the two surfaces to be joined, knotting each stitch in the centre.

\subsection{Continuous suture (Type 2)}

The entire suture is completed with the same thread, creating stitches that are perpendicular to the two surfaces to be joined, but knotting only the first and the last.

\subsection{Proposed “double-diabolo" suture (Type 3)}

This technique is based on the idea that a single stitch can form the locus of eight 45-degree angles. We start with a central stitch that is perpendicular to the surfaces to be joined (Fig. 2A). We then create an X-shaped stitch to the right (Figs. 2B and 2C), return with a second central stitch (Fig. 2C), create another X-shaped stitch, this time to the left (Figs. 2D and 2E), and finally return with a third central stitch to complete the "central column" (Fig. 2E). The stitch assembly is then knotted with the thread that was centrally located at the outset (Fig. 2F).

\section{Results}

\subsection{Type 1. Thread aligned with the force applied}

Three stitches are inserted in the same direction as the force applied (Fig. 3), by threads uniformly distributed along the probe.

The tension exerted on each thread Ft can be calculated, assuming that the system is in equilibrium, i.e. ? $\mathrm{F}=0$, and that this tension is applied equally over the entire system.

The external force, $F$, is distributed equally among the threads, i.e. F1=F2=F3 = F/3. The axial tension is exerted equally on each thread, i.e. $\mathrm{Ft}=\mathrm{F} / 3$.

The resulting tension exerted on each stitch is also equally distributed, i.e. Fs=F/3. 


\subsection{Type 2. Thread inserted in a zigzag trajectory}

In this method, only two stitches are inserted. The thread is guided along both sides of the wound, in a zigzag trajectory forming an angle, 0 , calculated as tan $0=\mathrm{b} / \mathrm{a}$ (Figure 4).

In this case, a single thread is inserted, and the axial force exerted on the thread, $\mathrm{Ft}$, is the same at every point. This force is calculated by applying the equilibrium equation to the system along the $\mathrm{x}$ and $\mathrm{y}$ axes. Figure 5 shows the forces exerted at each entry/exit point.

These points are not all subjected to the same level of tension, as less force is applied to the points on the right side of the wound than to those on left. Moreover, the tension exerted on the left and centre points also has an $\mathrm{x}$-axis component, which may provoke tearing in that direction.

The tension exerted on each thread, $\mathrm{Ft}$, is calculated as follows, assuming that the system is in equilibrium, i.e. 마 $\mathrm{F}=0$, and that the tension is applied on the y axis

$F_{1}+F_{2} \cos \alpha+F_{3}+F_{4} \cos \alpha+F_{3}=F$

$F_{1}=F_{2}=F_{3}=F_{4}=F_{t}$, and therefore

$F_{t}(3+2 \cos \alpha)=F \rightarrow F_{t}=\frac{F}{3+2 \cos \alpha}$

where Ft is the mechanical stress exerted on the thread.

The force exerted on each stitch and entry/exit point, FR, is also calculated when both forces are addressed, taking each force as a vector with its corresponding magnitude and direction

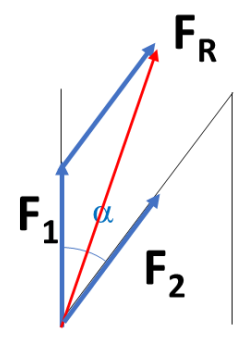

$$
\overrightarrow{F_{R}}=\overrightarrow{F_{1}}+\overrightarrow{F_{2}}
$$

By projecting the forces on the $\mathrm{x}$ and $\mathrm{y}$ axes, the following results are obtained

$F_{y}=F_{1}+F_{2} \cos \alpha=F_{t}(1+\cos \alpha)$

$F_{x}=F_{2} \sin \alpha=F_{t} \sin \alpha$

The magnitude of the resulting force can be calculated from the expression

$\left|\overrightarrow{\boldsymbol{F}_{\boldsymbol{R}}}\right|=\sqrt{F_{x}^{2}+F_{y}^{2}}=\mathbf{F}_{\mathrm{t}} \sqrt{2+2 \cos \alpha}=\frac{F \sqrt{2+2 \cos \alpha}}{3+2 \cos \alpha}$

This force is exerted on the first two entry/exit points; the third is only subjected to $\mathrm{Ft}$, and thus receives less mechanical stress.

\subsection{Type 3. Thread inserted in a double-cross pattern}

The aim of this suturing technique is to reduce the concentration of tensions exerted on the entry/exit points. This is achieved as follows. The thread is inserted along both sides of the wound, forming a double-cross pattern with an angle, ], calculated as $\tan 0=\mathrm{b} / \mathrm{a}$ (Figure 6). 
In this case, too, a single thread is inserted, in line with the probe, such that a constant axial force, $\mathrm{Ft}$, is exerted on the thread. To calculate Ft the equilibrium equation is applied to the system along the $\mathrm{x}$ and $\mathrm{y}$ axes. Figure 7 shows the forces applied at each entry/exit point.

The tension exerted by each thread, $\mathrm{Ft}$, is calculated from the equilibrium equation $\mathrm{F} \mathrm{F}=0$, applied to the $\mathrm{x}$ and $\mathrm{y}$ axes as follows

$F_{1}+F_{2} \cos \alpha+F_{3} \cos \sigma+F_{4}+F_{5} \cos \beta+F_{6}+F_{7}+F_{8} \cos \alpha+F_{9} \cos \sigma+F_{10}=F$

$F_{2} \sin \alpha+F_{5} \sin \beta-F_{3} \sin \sigma+F_{8} \sin \alpha-F_{9} \sin \sigma=0$

$F_{1}=F_{2}=F_{3}=F_{4}=F_{5}=F_{6}=F_{7}=F_{8}=F_{9}=F_{10}=F_{t}$, and therefore

$\mathrm{Ft}_{\mathrm{t}}(5+2 \cos \alpha+\cos \beta+2 \cos \sigma)=\mathrm{F} \rightarrow \mathrm{F}_{\mathrm{t}}=\frac{F}{5+2 \cos \alpha+\cos \beta+2 \cos \sigma}$

where Ft is the mechanical stress exerted on the thread.

The tension exerted on each stitch and entry/exit point, FR, is also assessed for the situation in which all the forces are taken into account. The resulting force is then calculated for each point, taking the force as a vector with the corresponding magnitude and direction

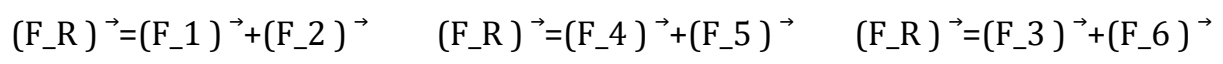
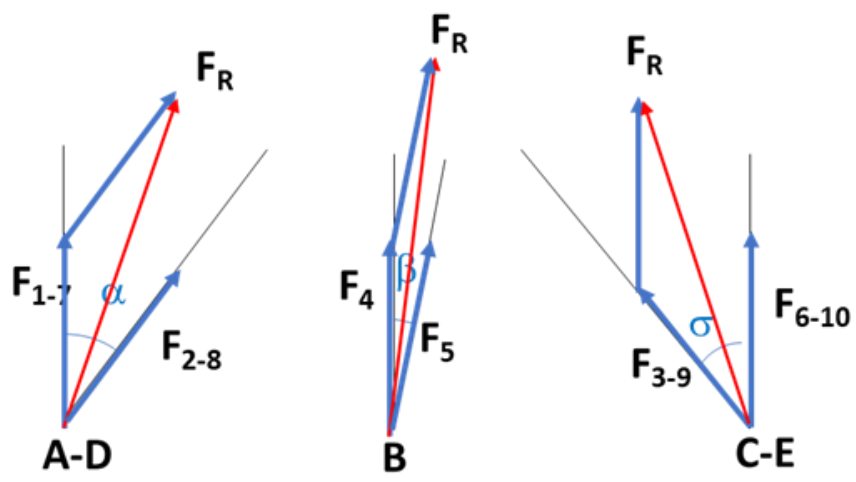

By considering the projection of the forces on the $\mathrm{x}$ and $\mathrm{y}$ axes, the following results are obtained for each entry/exit point:

Points A and D

$F_{x}=F_{1}+F_{2} \cos \alpha=F_{t}(1+\cos \alpha)$

$F_{y}=F_{2} \sin \alpha=F_{t} \sin \alpha$

The magnitude of the resulting force is calculated from the expression:

$\left|\overrightarrow{F_{R}}\right|=\sqrt{F_{x}^{2}+F_{y}^{2}}=F_{t} \sqrt{2+2 \cos \alpha}=\frac{F \sqrt{2+2 \cos \alpha}}{5+2 \cos \alpha+\cos \beta+2 \cos \sigma}$

Point B

$F_{x}=F_{6}+F_{6} \cos \beta=F_{t}(1+\cos \beta)$

$F_{y}=F_{6} \sin \beta=F_{t} \sin \beta$

The magnitude of the resulting force is calculated from the expression: 
$\left|\overrightarrow{F_{R}}\right|=\sqrt{F_{x}^{2}+F_{y}^{2}}=\mathbf{F}_{\mathrm{t}} \sqrt{2+2 \cos \beta}=\frac{F \sqrt{2+2 \cos \beta}}{5+2 \cos \alpha+\cos \beta+2 \cos \sigma}$

Points $C$ and $E$

$F_{x}=F_{1}+F_{2} \cos \sigma=F_{t}(1+\cos \sigma)$

$F_{y}=-F_{2} \sin \sigma=-F_{t} \sin \sigma$

The magnitude of the resulting force is calculated from the expression:

$\left|\overrightarrow{F_{R}}\right|=\sqrt{F_{x}^{2}+F_{y}^{2}}=\mathbf{F}_{\mathrm{t}} \sqrt{2+2 \cos \sigma}=\frac{F \sqrt{2+2 \cos \sigma}}{5+2 \cos \alpha+\cos \beta+2 \cos \sigma}$

The forces exerted on all the points in this group are not balanced in the $\mathrm{x}$ axis and will tend to tear the point in the direction of the resulting force.

Once the main equations have been obtained for each group, the results can be compared in terms of the force exerted on the thread and on each entry/exit point and stitch.

The following results highlight the differences between the three suture methods when a force of $10 \mathrm{~kg}$ is applied. The geometrical relations for the parameters in each case are

- $\mathrm{a}=1 \mathrm{~cm}$

- $b=1 \mathrm{~cm}$

- $\mathrm{d}=0.2 \mathrm{~cm}$

- $\alpha=45^{\circ}$

- $\beta=11.31^{\mathrm{o}}$

- $\sigma=47.73^{\circ}$

Table 1 show the results obtained, for each of the suturing methods considered. The lowest level of thread tension was recorded for Type 3 (almost 65\% less than that for Type 1 and 50\% less than that for Type 2). The Type 3 method also produced least tension on the suture exit/entry points (almost 33\% less than for Type 1 and 50\% less than for Type 2).

Table 1 Theoretical results obtained for the three suturing methods

\begin{tabular}{|l|l|l|}
\hline & $\begin{array}{l}\mathbf{F}_{\mathbf{t}}[\mathbf{k g}] \\
\text { (force on the thread) }\end{array}$ & $\begin{array}{l}\mathbf{F}_{\mathbf{R}} \text { [kg] } \\
\text { (force on entry/exit points) }\end{array}$ \\
\hline Group 1. Thread aligned with force applied & $3.33 \mathrm{~kg}$ & $3.33 \mathrm{~kg}$ \\
\hline Group 2. Thread applied in a zigzag trajectory & $2.26 \mathrm{~kg}$ & $\begin{array}{l}4.17 \mathrm{~kg} \text { (points 1 and 2) } \\
2.26 \text { (point 3) }\end{array}$ \\
\hline $\begin{array}{l}\text { Group 3. Thread applied in a doble x-shaped pattern, } \\
\text { though various entry/exit points }\end{array}$ & $1.144 \mathrm{~kg}$ & $\begin{array}{l}2.11 \mathrm{~kg} \text { (points A and D) } \\
2.27 \mathrm{~kg} \text { (point B) } \\
2.09 \mathrm{~kg} \text { (points C and E) }\end{array}$ \\
\hline
\end{tabular}

\section{Discussion}

The insertion of sutures is a surgical task that is both challenging and laborious. Somewhere between 50,000 and 30,000 B.C. eyed needles were invented and by 20,000 B.c. bone needles were of a standard unsurpassed until the Renaissance. It is reasonable to assume that these needles were used to sew wounds together [1]. It is suggested that the original versions of suture threads were coarse and made from plant fibers before progressing to animal parts much later in history. In ancient India, Egypt and the Greek and Roman societies wound treatments as well as suturing techniques and instruments were developed that strongly resemble those in our days [2]. 
Despite the development of new suture materials and proposals for new suture techniques, there has been no significant improvement in the results obtained [7,8]. In several large randomized trials of more than 500 patients it has been shown that small bite size sutures (as first reported by Israelsson [9]) can reduce the development of incisional hernias $[10,11]$. Small bites have shown a higher bursting pressure than large bites: if the tension on the wound is distributed over a large number of stitches, the tension on each stitch will be low [12].

Computer models for surgical intervention simulation to improve the operation quality have began to be applied recently. Using the software, the surgeons may improve their technique or elaborate new methods for the patientspecific treatment [13-16]. Most papers are devoted to the soft tissue/medical tool interaction with haptic feedback to create VR simulator [16-20].

Shilko et al [21] obtained an analytical solution of the contact interaction problem of polymeric suture material with a bio-tissue. A pull-out test is analyzed to evaluate the stress intensity in the tissue. The paper contains an example of model application for skin wound suturing computations. Some paper devoted to tensile strength of sutures and knots can be highlighted to evaluate mechanical properties of sutures [22-25].

Evidently, the fundamental mechanisms governing the operation of living and non-living beings are subject to the laws of the pure sciences, i.e. physics, chemistry and mathematics, and surgical interventions are bound by the same laws ]26,27].

In this respect, Srivasgava [27] studied the application of the laws of mechanics, thermodynamics and vectors to the soft and bony tissues of the body. This study was based on a systematic search of the Medline database for the period 1960-2008, using keywords such as Mechanics, Laplace's law, Pascal's law, Vector laws and Suture techniques. The texts referred to Laplace's law in relation to a case of colon perforation, compression therapy, parturition, variceal rupture, disc herniations etc. The Pascal's Law finds use in hernia repair and the Heimlich maneuver. Trigonometrically derived components of forces, acting after suturing, show ways to reduce cut-through; the thickness and the bite of suture determines the extent of tissue reaction.

In the same study, Srivasgava discussed the fact that the tension exerted on surgical stitches decreased in accordance with the cosine of the angle presented. However, no specific proposal was made to take advantage of this physical law.

Despite the technical advances that have been made with sutures, our review of the literature did not reveal any prior detailed study of the physical laws governing the tensions generated on threads and sutures, seeking to optimise the materials and method used. In fact, the techniques used remain largely unchanged, i.e. the sutures created are either continuous or interrupted (with minor variations). In other words, the stitches created are perpendicular to the tensile forces present and are fully exposed to the tension exerted on the suture thread.

Therefore, although the advances of modern science have greatly improved the materials employed, suturing techniques as such have not progressed at all, in thousands of years. Nevertheless, a fresh approach is needed, in order to achieve two crucial aims: on the one hand, to prevent the wound tissues from tearing at the puncture sites, and on the other, to disperse the forces produced, along the entire suture line, so that each thread supports the least possible tension.

To achieve these goals, we take into account the force vectors acting on a suture. In this respect, the tension exerted on a thread that joins two divergent points decreases in accordance with the cosine of the angle formed by the thread and the perpendicular to the force applied to separate the points; in other words, the more points at which the thread is anchored, the less tension is exerted upon it [27]. For example, for an angle of 45 degrees, the corresponding cosine is 0.7 . Therefore, the tension on the thread is the force administered multiplied by 0.7 (i.e., this force is reduced by $30 \%$ ). The suture techniques currently used, whether interrupted or continuous, join points in a straight line; hence, the angulation is zero and the sutures must withstand the maximum possible tension.

In view of these considerations, and after assessing many possible suture structures, we propose a new suture design (the one that produced the best results in the theoretical model), with four 45-degree angles at each point of the surfaces to be joined. In total, there are eight angles, emerging from a triple-column central structure.

A notable finding of the present study is the inferior performance of the continuous suture method, according to our theoretical model. The tension generated in these sutures includes a lateral element at each point, equivalent to the vertical force applied at the cosine of the angle created, which can cause lateral tears, and thus destabilise the suture. With the new method proposed, the lateral angles must also withstand tension. However, as the vertical force applied 
is only half that exerted on the continuous suture, when this force is multiplied by the cosine of the angle, the resulting lateral force exerted on each entry/exit point is also half that of the continuous suture. Therefore, the suture is twice as resistant to lateral tearing.

A weakness in the proposed suture might be observed if the three central stitches were passed through the same entry/exit point, where the theoretical model predicts a tension of $5.63 \mathrm{~kg}$. To overcome this problem, in the final version of the model we use a pattern of separated central points, which reduces the tension exerted on each point to just over $2 \mathrm{~kg}$.

In summary, the results obtained in this study corroborate our hypothesis that the "double-diabolo" suturing method reduces the tension exerted on the thread and the entry/exit points (for a given separation force), in comparison with conventional techniques.

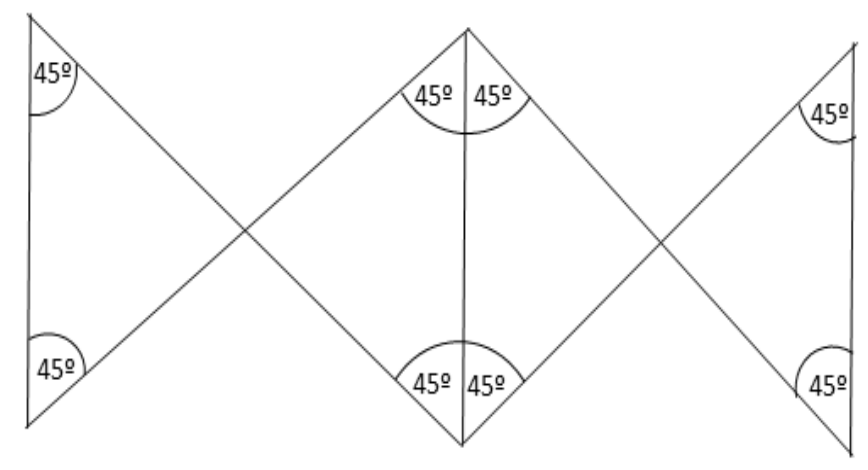

Figure 1 Theoretical model to reduce tension via an 8-angle suture pattern.

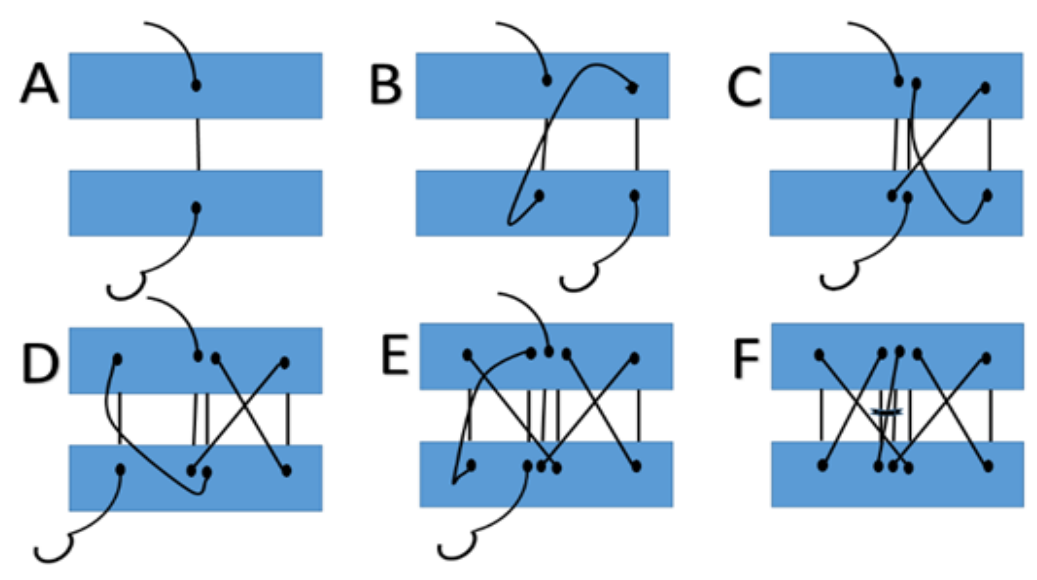

Figure 2 Steps to create the proposed "double-diabolo" suture. 

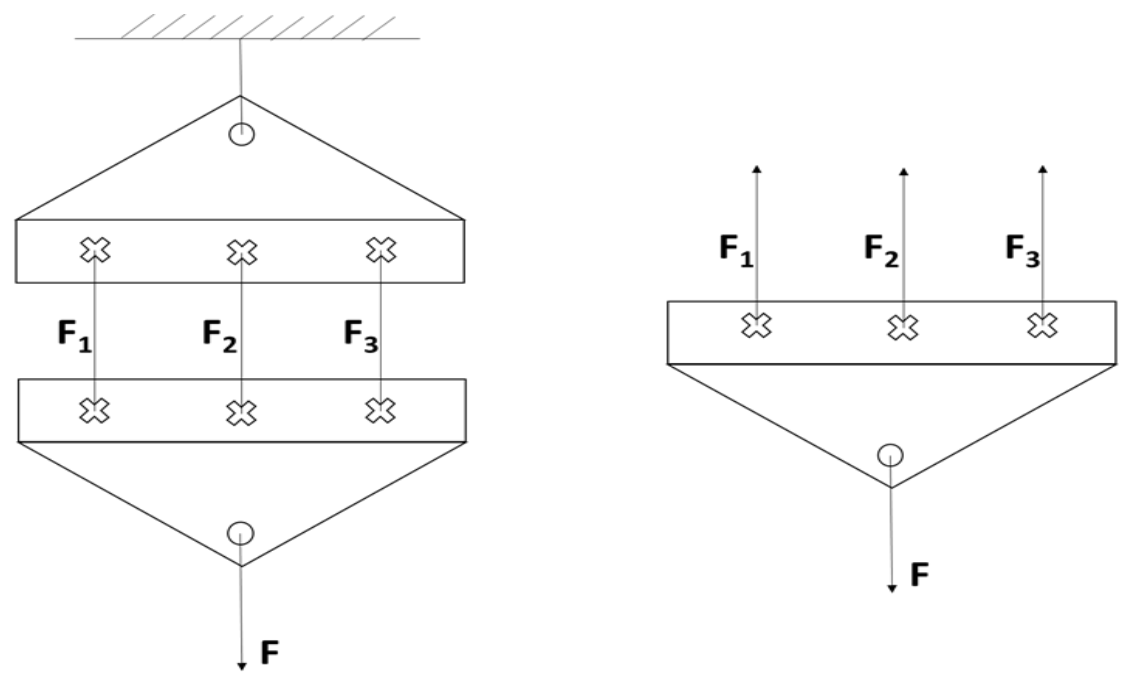

Figure 3 Aligned thread and distribution of forces.
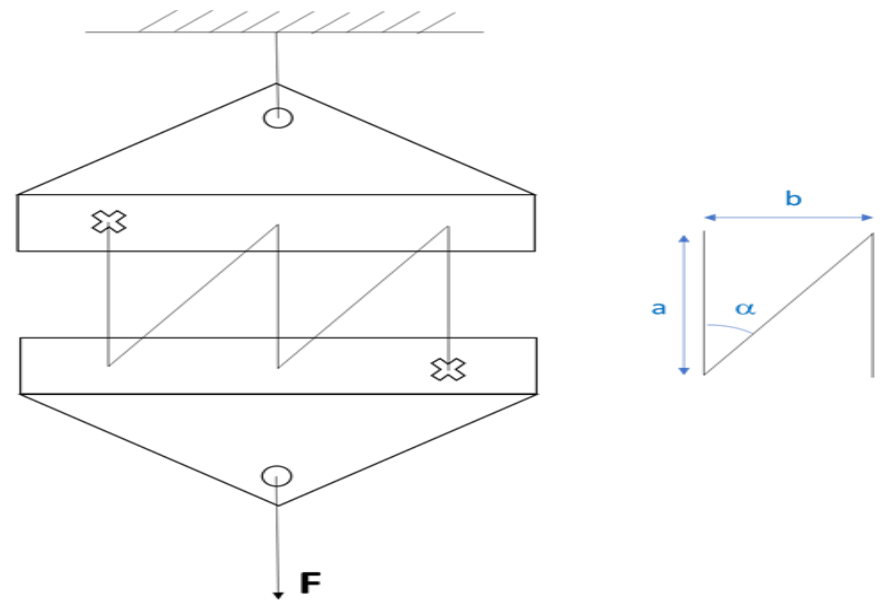

Figure 4 Thread applied following a zigzag trajectory.
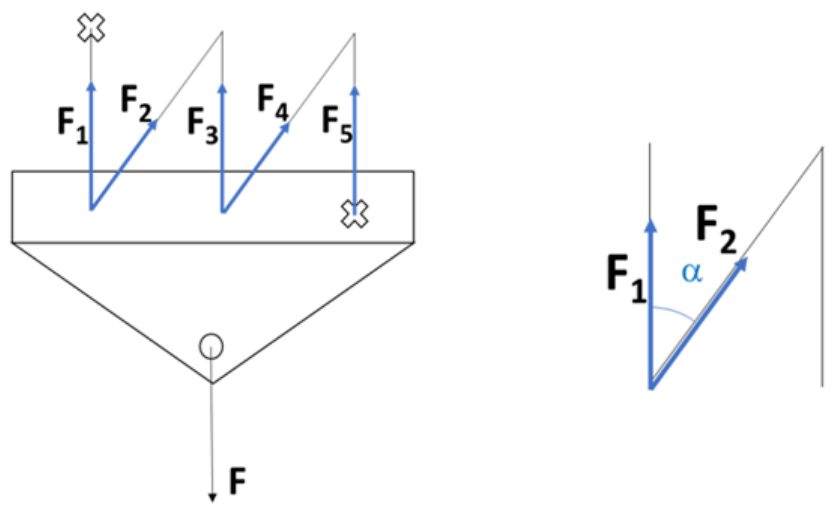

Figure 5 Distribution of axial forces. 

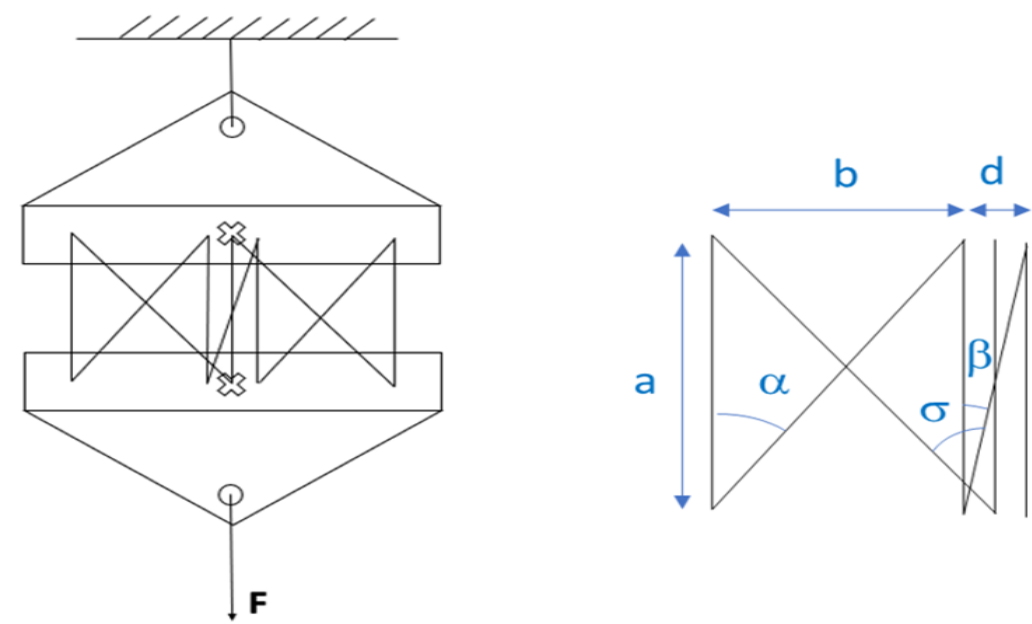

Figure 6 Thread applied in a double x-shaped pattern, though various entry/exit points.
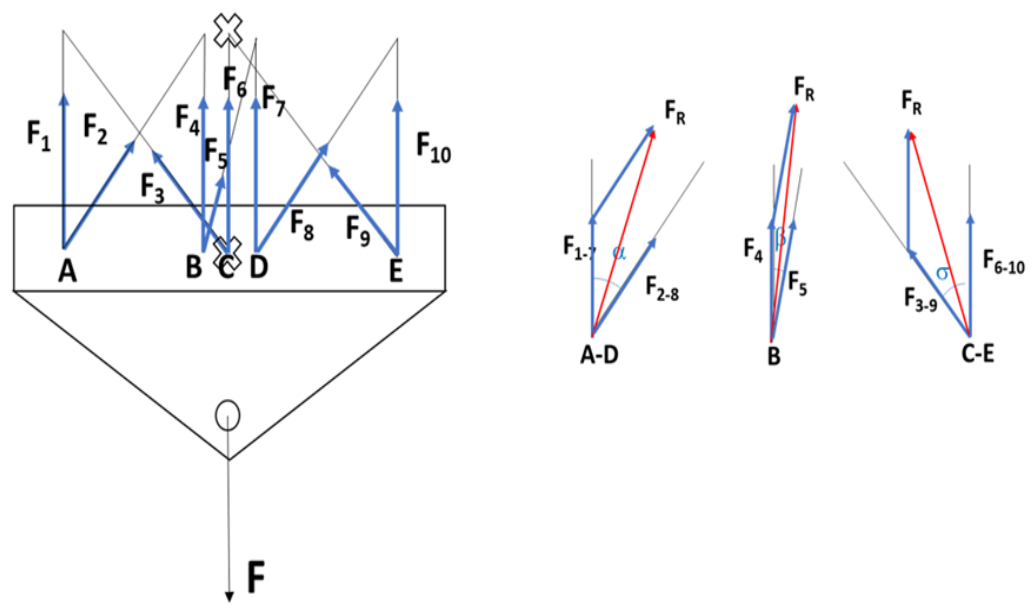

Figure 7 Distribution of axial forces.

\section{Conclusion}

We believe that if definitive results of phase two and three of the study confirm theoretical results the suturing technique described is appropriate for many medical situations, especially when the suture must withstand significant tension, in actions such as laparotomy closure, thoracotomy closure, diaphragm suturing or hernia orifice closure.

When phases two and three of the study are complete, we shall publish our definitive findings. However, the preliminary results obtained in practice (phase two and three), appear to corroborate the conclusions drawn from the theoretical model.

\section{Compliance with ethical standards}

\section{Disclosure of conflict of interest}

The corresponding authors have no conflicts of interest. 


\section{References}

[1] Kirkup J. The Evolution of Surgical Instruments; An Illustrated History from Ancient Time to the Twentieth Century. 176.

[2] Kuijjer PJ. History of healing; wound suturing. Ned Tijdschr Geneeskd. 1998; 142(9): 473-9.

[3] Högström H, Haglund U, Zederfeldt B. Suture technique and early breaking strength of intestinal anastomoses and laparotomy wounds. Acta Chir Scand. 1985;151: 441-443.

[4] Högström H, Haglund U, Zederfeldt B. Tension leads to increased neutrophil accumulation and decreased laparotomy strength. Surgery. 1990; 107: 215-219.

[5] Israelsson LA. The surgeon as a risk factor for complications of midline incisions. Eur J Surg. 1998; 164: 353-357.

[6] Trimbos JB, van-Rooij J. Amount of suture material needed for continuous or interrupted wound closure: an experimental study.Eur J Surg. 1993; 159: 141-143.

[7] Bloemen A, Van Dooren P, Huizinga BF, Hoofwijk AG. Randomized clinical trial comparing polypropylene or polydioxanone for midline abdominal wall closure. Br J Surg. 2011; 98(5): 633-9.

[8] Fink C, Baumann P, Wente MN, Knebel P, Bruckner T, Ulrich A, et al. Incisional hernia rate 3 years after midline laparotomy. Br J Surg. Jan 2014; 101(2): 51-4.

[9] Israelsson LA, Millbourn D. Closing midline abdominal incisions. Langenbecks Arch Surg. 2012; 397(8): 12011207.

[10] Deerenberg EB, Harlaar JJ, Steyerberg EW, Lont HE, van Doorn HC, Heisterkamp J et al. Small bites versus large bites for closure of abdominal midline incisions (STITCH): a double-blind, multicentre, randomised controlled trial. Lancet. 2015; 386(10000): 1254-1260.

[11] Millbourn D, Cengiz Y, Israelsson LA. Effect of stitch length on wound complications after closure of midline incisions: a randomized controlled trial. Arch Surg. 2009; 144(11): 1056-1059.

[12] Cengiz Y, Blomquist P, Israelsson LA. Small tissue bites and wound strength: an experimental study. Arch Surg. 2001; 136(3): 272-5.

[13] Lian LL, Chen YH. Haptic surgical simulation: an application to virtual suture, Computer-Aided Design \& Applications. 2006; 3(1-4): 203-210.

[14] Aloisio G, Tommaso L, Mongelli A, Provenzano L. Artery soft-tissue modelling for stent implant training system, Systemics, cybernetics and informatics. 2002; 2(4): 7-11.

[15] Hao L. Predictive surgical simulation for cardiac surgery, Journal of Biomechanics. 2008; 14: 1-53.

[16] Jones IA, Becker AA, Glover AT, Wang P, Benford SD, Vloeberghs M et al. Computational mechanics in virtual reality: cutting and tumour interactions in a boundary element simulation of surgery on the brain, Journal of Biomechanics. 2005; 38: 1-8.

[17] De Novi G, Melchiorri C. Surgery simulations and haptic feedback: a new approach for local interaction using implicit surfaces, Proceedings of International Conference on Applied Bionics and Biomechanics, (14- 16 October 2010, Venice, Italy), Italy. 2010.

[18] Lima M, Melchiorri C, Ruggeri G, De Novi G, Gargano T, Mogiatti M et al. A new robotic platform for endoscopic skill training, Proceedings of 17 International Congress on Endoscopic Surgery (17-20 June 2009, Prague, Czech Republic), Springer. 2009.

[19] Bro-Nielsen M, Finite element modeling in surgery simulation, Proceedings of the IEEE, 1998;86(3):490-503.

[20] Picinbono G, Delingette H, Ayache N. Nonlinear anisotropic elasticity for real-time surgery simulation, Graphical Models. 2003; 65(5): 305-321.

[21] Shilko SV, Chernous DA, Panin SV. Model and analysis of contact interaction of one-dimensional polymeric implants with biotissues. Russian Journal of Biomechanics. 2011; 15(1): 65-73.

[22] Pgez GJM, San Martin CA, Sestafe AG, Herrero EG, Navidad R, Cordon A et al. Elastic behaviour of sutured calf pericardium: influence of the suture threads, Biomaterials. 1996; 17: 1677-1683.

[23] Nishimura KA, Mori R, Miyamoto W, Uchio Y. New technique for small and secure knots using slippery polyethylene sutures, Clinical Biomechanics. 2009; 24: 403-406. 
[24] Balgobin S, Hamid CA, Brown SA, Wai CY. Mechanical performance of surgical knots in a vaginal surgery model, Journal of Surgical Education. 2013; 70(3): 340-344.

[25] van't Riet M, de Vos van Steenwijk PJ, Kleinrensink GJ, Steyerberg EW, Bonjer HJ. Tensile strength of mesh fixation methods in laparoscopic incisional hernia repair. Surg Endosc. 2002; 16(12): 1713-1716.

[26] Anurag S, Akshay S, ParijatJoy S, John W. Principles of physics in surgery: the laws of flow dynamics physics for surgeons-part 1. Indian J Surg. 2009; 71: 182-187.

[27] Srivastava A, Sood A, Joy PS, Mandal S, Panwar R, Ravichandran S et al. Principles of Physics in Surgery: The Laws of Mechanics and Vectors Physics for Surgeons-Part 2. Indian J Surg. 2010; 72(5): 355-361. 